\title{
Miscarriage Occurrence and Prevention Efforts by Disability Status and Type in the United States
}

\author{
Mekhala V. Dissanayake, MPH, Blair G. Darney, PhD, MPH, ${ }^{1-3}$ \\ Aaron B. Caughey, MD, PhD, ${ }^{1}$ and Willi Horner-Johnson, $\mathrm{PhD}^{2,4}$
}

\begin{abstract}
Background: Very little is known about early pregnancy loss in women with disabilities. To address this gap, we analyzed nationally representative data on miscarriage and receipt of care focused on miscarriage prevention among women with and without disabilities.

Materials and Methods: We used 2011-2015 National Survey of Family Growth data on women with at least one completed pregnancy within the past 5 years. Bivariate and multivariate analyses assessed the association of six disability categories (any, hearing, vision, cognitive, physical, independent living) with miscarriage occurrence and receipt of services intended to prevent miscarriage.

Results: Our analytic sample included 3,843 women with 5,776 completed pregnancies within the past 5 years. Overall, $31.63 \%$ of women with disabilities and $21.83 \%$ of women without disabilities had had a miscarriage within the past 5 years. Compared to women without disabilities, women with any, cognitive, physical, and independent living disability had higher adjusted odds of experiencing miscarriage (any disability aOR $=1.65$ [95\% CI: 1.21-2.25]). These women also had higher odds of receiving services to prevent miscarriage compared with women without disabilities (any disability aOR $=1.71$ [95\% CI: 1.20-2.45]). Among women who received services, higher proportions of women with any, vision, physical, or independent living disability received recommendations for bed rest (e.g., 65.007\% of women with independent living disability vs. $33.98 \%$ of women without disability, $p=0.018)$.

Conclusions: In a representative sample of U.S. women, we found significant differences in the odds of miscarriage and in receipt of care to prevent miscarriage between women with and without disabilities. Further research is needed to understand why women with disabilities are more likely to experience a miscarriage. Such research is important for informing care recommendations.
\end{abstract}

Keywords: persons with disabilities, miscarriage, obstetrics, reproductive health

\section{Introduction}

W OMEN WITH DISABILITIES experience pregnancy at rates similar to women without disabilities. ${ }^{1,2} \mathrm{Re}$ search to date has demonstrated disparities for women with disabilities in prenatal care utilization and satisfaction with prenatal care, preterm birth, having infants small for gestational age, and high rates of cesarean deliveries. ${ }^{3-19}$ The majority of this research is based on delivery records or surveys like the Pregnancy Risk Assessment Monitoring
System (PRAMS), which contain only live birth outcomes and therefore do not reflect early pregnancy loss or miscarriage. Thus, considerably less is known about the association between disability and miscarriage. One prior study found that women with complex disabilities (those that affected activities of daily living and/or instrumental activities of daily living) were at greater risk of miscarriage than women without disabilities. ${ }^{20}$ However, miscarriages among women with different types of disabilities (physical, cognitive, sensory) have not yet been examined.

Preliminary versions of some of the findings reported in this article were presented at the AcademyHealth Annual Research Meeting in Seattle, WA; June 2018 and at the Western Institute for Nursing Research Conference in San Diego, CA; April 2019.

${ }^{1}$ Department of Obstetrics \& Gynecology, Oregon Health \& Science University, Portland, Oregon.

${ }^{2}$ School of Public Health, Oregon Health \& Science University/Portland State University, Portland, Oregon.

${ }^{3}$ National Institute of Public Health (INSP), Center for Population Health Research (CISP), Cuernavaca, Mexico.

${ }^{4}$ Institute on Development and Disability, Oregon Health \& Health University, Portland, Oregon. 
Miscarriage is considered the most common pregnancy complication, with clinically recognizable miscarriage occurring in $12 \%-15 \%$ of pregnancies, though the rate of loss between implantation and clinical identification is likely closer to $50 \% .^{21}$ Because miscarriage is relatively common and most frequently related causally to aneuploidy, clinicians generally focus on preventing recurrent miscarriage, which affects about $1 \%$ of couples. ${ }^{22}$ Recurrent miscarriage is formally defined as three or more consecutive pregnancy losses, though in practice, clinicians may intervene after two miscarriages due to patient concern. ${ }^{23}$ There is no currently existing research on the extent to which women with disabilities experience recurrent miscarriages or receive medical care intended to prevent miscarriages.

The purpose of this study was to determine (1) whether occurrence of miscarriage differs by disability status and type; (2) whether women with disabilities are more likely to have recurrent miscarriages; and (3) what care, if any, women with disabilities have received to prevent miscarriage.

\section{Materials and Methods}

\section{Data source}

We used the combined 2011-2013 and 2013-2015 waves of the National Survey of Family Growth (NSFG). The NSFG is designed and administered by the National Center for Health Statistics (NCHS) to produce data on sexual activity, marriage, divorce, pregnancy, contraceptive practices, and other aspects of reproductive health in the United States. ${ }^{24}$ Interviews are administered in person using a combination of computer-assisted personal interviewing and audio computerassisted self-interviewing. The survey employs a continuous sampling design over a 2-year period to obtain nationally representative samples of women and men of reproductive age (15-44 years) in civilian households. The weighted response rates for women were $73.4 \%$ for $2011-2013$ and $71.2 \%$ for $2013-2015 .^{25}$

The NSFG provides data from women in two files. The female respondent file includes sociodemographic characteristics of each woman at the time of interview, along with information about her sexual and contraceptive history. A separate pregnancy file contains retrospective data about each pregnancy reported by each woman. The pregnancy file contains zero, one, or multiple records for each female respondent, depending on how many pregnancies she has had. We merged data from the NSFG pregnancy files with the female respondent files to create a single data file that allowed us to determine the disability status of the woman associated with each individual pregnancy.

\section{Analytic sample}

Our analysis included women in the NSFG survey who had reported at least one pregnancy in the last 5 years and had nonmissing data on disability status and the covariates described below. While information on lifetime pregnancies and miscarriages was available, the NSFG does not provide any data on duration of disability. Thus, we were not able to determine whether or not women had disabilities at the time of their pregnancies. To reduce the likelihood of including pregnancies that may have occurred before disability onset, we restricted our analyses to pregnancies completed within the 5 years before interview. We excluded current pregnancies and ectopic pregnancies.

\section{Dependent variables}

We analyzed four dependent variables with women as the unit of analysis. These first two of these variables were: whether women had any pregnancy ending in miscarriage in the past 5 years (yes/no); and whether women had two or more pregnancies ending in miscarriage in the past 5 years (yes/no), based on responses in the pregnancy file. The third and fourth variables with women as the unit of analysis utilized responses to a survey question in the respondent file that asked whether the participant had received any medical help to prevent miscarriage. We analyzed: whether women had ever received any services intended to prevent miscarriage (yes/no); and, if so, the type of miscarriage prevention services received. Among women who received miscarriage prevention services, we assessed the proportions who received the most common services: diagnostic testing; administration of progesterone or synthetic progestogen; bed rest; restricted physical activity.

We analyzed two additional variables with pregnancies as the unit of analysis: (1) proportion of pregnancies ending in miscarriage; and (2) at what week of pregnancy loss occurred (if applicable). The specific samples used for each research question are summarized in Figure 1.

\section{Primary independent variables}

We categorized disability status and type based upon responses to six questions used by the U.S. Census Bureau since 2008 and adopted in 2011 by the Department of Health and Human Services as the minimum standard for disability data collection in population-based health surveys. ${ }^{26}$ Beginning in 2011, all NSFG respondents were asked the following questions about disability:

(1) Do you have serious difficulty hearing? (Hearing disability)

(2) Do you have serious difficulty seeing, even when wearing glasses or contact lenses? (Vision disability)

(3) Because of a physical, mental, or emotional condition, do you have serious difficulty concentrating, remembering or making decisions? (Cognitive disability)

(4) Do you have serious difficulty walking or climbing stairs? (Mobility disability)

(5) Do you have difficulty dressing or bathing? (Self-care disability)

(6) Because of a physical, mental, or emotional condition, do you have difficulty doing errands alone such as visiting a doctor's office or shopping? (Independent living disability)

We classified women as having a given disability if they answered affirmatively to the corresponding question; thus a woman could be included in more than one disability category if she answered yes to more than one question. Because the number of women with difficulty dressing or bathing was quite small (1\% of the sample) and $80 \%$ of women with this disability also reported mobility difficulty (a greater degree 


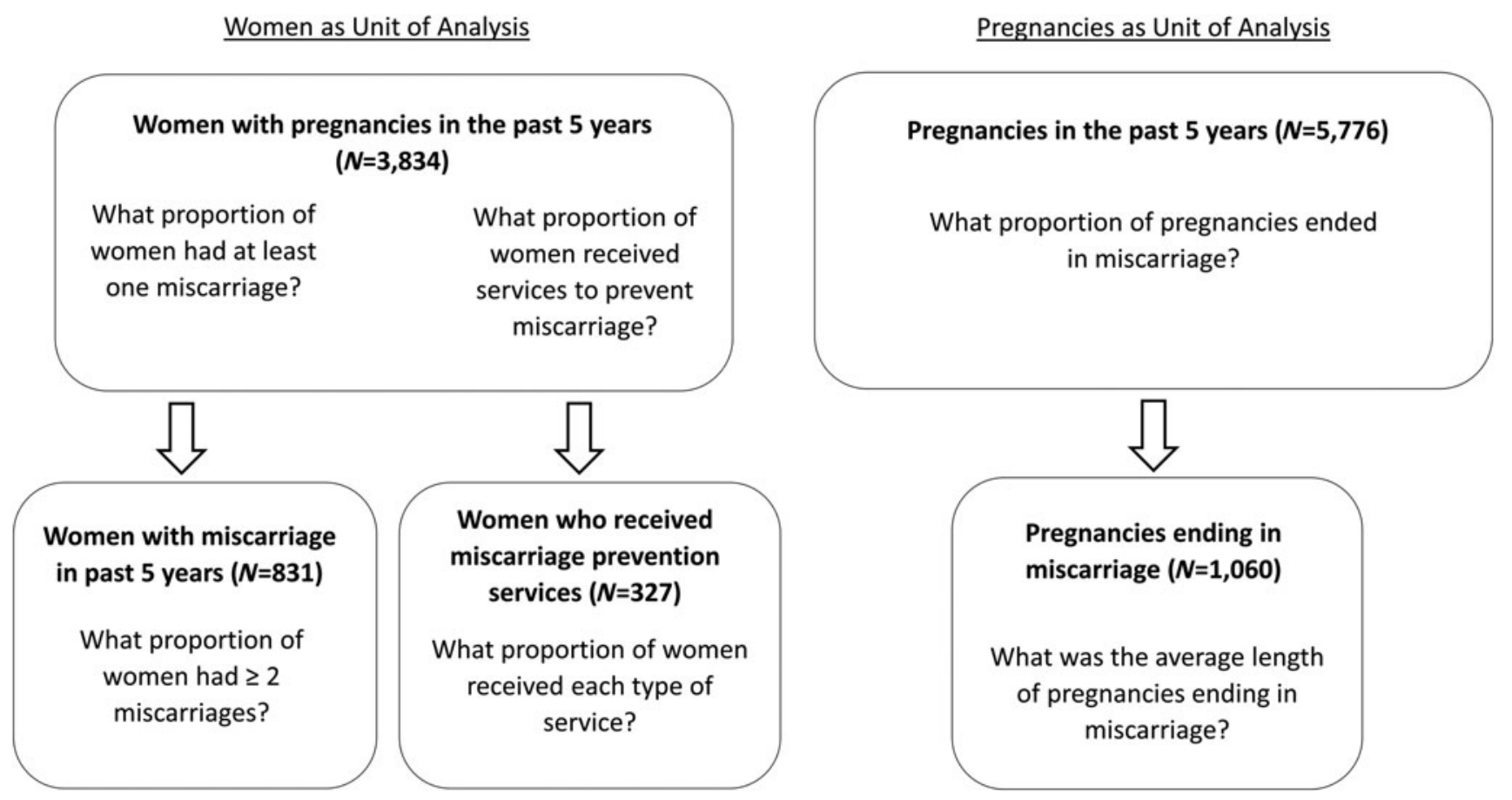

FIG. 1. Study samples (from National Survey of Family Growth, 2011-2015) and corresponding research questions.

of overlap than existed between any other two disability variables), we combined self-care disability and mobility disability to create a "physical disability" variable.

The comparison group of women without disabilities consisted of women who answer no to all six disability questions. We also created an overall disability variable that classified women as having any disability versus no disability. Thus, we analyzed findings for six disability groups: any disability, hearing disability, vision disability, cognitive disability, physical disability, and independent living disability.

\section{Covariates}

We adjusted for the following sociodemographic characteristics in our multivariate regression models: maternal age (15-24, 25-34, 35-44); race (non-Hispanic White, nonHispanic Black, Hispanic of any race, Other); current partner status (currently married or cohabitating versus not); education (college or more versus less than college); health insurance (privately insured, publicly insured, uninsured), parity (multiparous vs. nulliparous); and miscarriage history before the past 5 years. We used current partner status as a proxy for partner status during the last 5 years because preliminary analysis of variables on lifetime partners indicated that most women who were currently married or cohabitating at time of interview were also married or cohabitating during the last 5 years.

\section{Statistical analysis}

We conducted cross-sectional analyses of the combined 2011-2013 and 2013-2015 waves of NSFG data. For each of our six disability variables, we conducted chi-squared tests comparing the proportions of women with and without disabilities who had the following: (1) any miscarriages within the past 5 years; (2) more than one miscarriage in the past 5 years (among those who had miscarried); (3) received any services to prevent miscarriage. Among those who received services, we compared the proportions of women with and without disabilities who said they had experienced at least one pregnancy loss before receiving services, and the proportions receiving each type of service.

Additionally, we were interested in determining whether women with disabilities had more miscarriages because they had more pregnancies in the study period. To address this, we used chi-squared tests to compare the proportions of pregnancies that ended in miscarriage in each disability group to the proportion in the comparison group of women with no disabilities. We used $t$-tests to compare mean length (in weeks) of pregnancies ending in miscarriage among women in each disability group versus the comparison group of women with no disabilities.

We used multivariable logistic regression models, with women as the unit of analysis, to assess the adjusted association between the disability categories and (1) miscarriage in the last 5 years; and (2) receipt of services to prevent miscarriage. Our models adjusted for the covariates described above. We conducted six sets of regression models: one comparing women with any disability to women with no disability, and five comparing women in each of our five disability categories (one set of models per disability category) to women with no disability. We conducted all analyses with Stata 15, using the survey functions to account for the complex sampling design of the NSFG. We weighted our analyses - applying the 201120154 year weights provided by NCHS — to represent the U.S. population of reproductive age women.

\section{Results}

The combined 2011-2015 file included 11,300 women between the ages of 15 and 44. After applying our inclusion criterion of having a pregnancy within the last 5 years, 3,843 
women with a total of 5,776 pregnancies were included in this analysis (Fig. 1). Only four women with pregnancies in the last 5 years had missing information on disability status, and all women had complete information for the selected covariates. Sample sizes and sociodemographic characteristics for each disability group are presented in Table 1. Overall, women with disabilities were younger, more likely to be nulliparous, and less likely to be partnered, have a college education, or be privately insured. Women with physical disabilities and women with independent living disabilities were also more likely to have a prior history (longer than 5 years ago) of miscarriage.

With the exception of women with difficulty seeing, a higher proportion of women in each disability category had at least one pregnancy end in miscarriage in the last 5 years, compared with women without a disability (Table 2). This was most notable in women with difficulty hearing, who were almost twice as likely to have had a miscarriage as women with no disability $(41.66 \%$ vs. $21.83 \%, p=0.0041)$. Among women who experienced a miscarriage, we found that only women with independent living disability were significantly more likely to have experienced two or more miscarriages compared to women without disabilities (28.86\% vs. $16.13 \%$, $p=0.0488)($ Fig. 2).
Larger proportions of women with physical disability and women with independent living disability received services to prevent miscarriage compared to women with no disabilities (Table 2). We found that $65 \%$ of women who received services had already experienced at least one pregnancy loss; this percentage did not differ between women with any disability and women without disabilities $(65 \%$ for each). We were underpowered to assess whether women with specific disability types were more or less likely to have had a pregnancy loss before receiving services to prevent miscarriage. Among women who received services to prevent miscarriage, women with physical disability were much more likely than women without disabilities to report that they were advised to go on bed rest $(67.47 \%$ vs. $33.98 \%, p<$ $0.001)$ or restrict their physical activity $(86.72 \%$ vs. $50.81 \%$, $p<0.001$ ).

Our findings with pregnancies as the unit of analysis mirrored those with women as the unit of analysis. In each disability category except vision disability, the percentage of pregnancies that ended in miscarriage was significantly higher relative to pregnancies among women with no disabilities (Fig. 3). For example, $30.38 \%$ of pregnancies among women with any disability ended in miscarriage compared to $18.33 \%$ of pregnancies among women without disabilities

Table 1. Sociodemographic Characteristics of Women with Pregnancies in the Last 5 Years ( $N=3,834)$

\begin{tabular}{|c|c|c|c|c|c|c|c|}
\hline & \multicolumn{2}{|c|}{$\begin{array}{l}\text { Disability } \\
\text { status }\end{array}$} & \multicolumn{5}{|c|}{$\begin{array}{c}\text { Disability } \\
\text { type }\end{array}$} \\
\hline & $\begin{array}{c}\text { No } \\
\text { disability } \\
(\mathrm{n}=3,089)^{\mathrm{a}} \\
(83.58 \%)\end{array}$ & $\begin{array}{c}\text { Any } \\
\text { disability } \\
(\mathrm{n}=745) \\
(16.42 \%)\end{array}$ & $\begin{array}{l}\text { Hearing } \\
(\mathrm{n}=96) \\
(2.84 \%)\end{array}$ & $\begin{array}{c}\text { Vision } \\
(\mathrm{n}=201) \\
(4.91 \%)\end{array}$ & $\begin{array}{c}\text { Cognitive } \\
(\mathrm{n}=489) \\
(11.48 \%)\end{array}$ & $\begin{array}{c}\text { Physical } \\
(\mathrm{n}=129) \\
(2.98 \%)\end{array}$ & $\begin{array}{c}\text { Independent } \\
\text { living } \\
(\mathrm{n}=144) \\
(2.98 \%)\end{array}$ \\
\hline \multicolumn{8}{|l|}{ Maternal age } \\
\hline $15-24$ & 18.95 & 29.93 & 25.79 & 31.49 & 32.11 & 13.54 & 31.89 \\
\hline $25-34$ & 52.98 & 49.04 & 48.76 & 46.93 & 47.20 & 54.46 & 43.97 \\
\hline $35-44$ & 28.07 & 21.02 & 25.45 & 21.58 & 20.70 & 32.00 & 24.14 \\
\hline$p$-value & Referent & $<0.001$ & 0.436 & 0.012 & $<0.001$ & 0.457 & 0.023 \\
\hline \multicolumn{8}{|l|}{ Race/ethnicity } \\
\hline White & 54.14 & 55.94 & 64.07 & 44.05 & 58.02 & 56.11 & 57.18 \\
\hline Black & 14.32 & 17.31 & 15.58 & 27.67 & 12.53 & 17.26 & 19.39 \\
\hline Hispanic & 23.34 & 22.69 & 16.78 & 24.79 & 24.11 & 21.82 & 19.04 \\
\hline Other & 8.20 & 4.06 & 3.57 & 3.49 & 5.44 & 4.81 & 4.00 \\
\hline$p$-value & Referent & 0.016 & 0.182 & $<0.001$ & 0.225 & 0.623 & 0.315 \\
\hline Partnered ${ }^{b}$ & 76.49 & 59.67 & 70.22 & 64.27 & 56.50 & 59.18 & 44.94 \\
\hline$p$-value & Referent & $<0.001$ & 0.250 & 0.004 & $<0.001$ & $<0.001$ & $<0.001$ \\
\hline College educated & 41.80 & 18.54 & 26.90 & 16.39 & 15.53 & 20.31 & 10.35 \\
\hline$p$-value & Referent & $<0.001$ & 0.049 & $<0.001$ & $<0.001$ & 0.003 & $<0.001$ \\
\hline \multicolumn{8}{|l|}{ Insurance } \\
\hline Private & 55.98 & 31.27 & 41.06 & 26.31 & 27.48 & 28.07 & 17.65 \\
\hline Public & 25.99 & 45.07 & 36.36 & 46.31 & 46.85 & 56.89 & 66.06 \\
\hline Other & 18.03 & 23.66 & 22.58 & 27.38 & 25.67 & 15.04 & 16.28 \\
\hline$p$-value & Referent & $<0.001$ & 0.183 & $<0.001$ & $<0.001$ & $<0.001$ & $<0.001$ \\
\hline Nulliparous & 8.47 & 13.31 & 19.00 & 13.13 & 13.67 & 8.56 & 13.71 \\
\hline$p$-value & Referent & $<0.001$ & 0.003 & 0.043 & 0.002 & 0.982 & 0.060 \\
\hline $\begin{array}{l}\text { Prior history of } \\
\text { miscarriage }\end{array}$ & 18.93 & 24.04 & 24.24 & 25.46 & 23.61 & 35.56 & 37.23 \\
\hline$p$-value & Referent & 0.039 & 0.333 & 0.106 & 0.098 & $<0.001$ & $<0.001$ \\
\hline
\end{tabular}

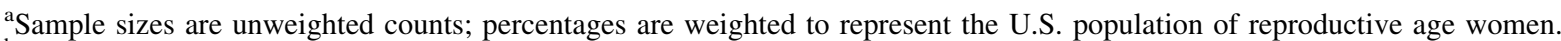
${ }^{\mathrm{b}}$ Partnered: Married or with a cohabiting partner.

${ }^{c}$ Prior history of miscarriage reflects any miscarriages that happened more than 5 years before interview.
} 
Table 2. Weighted Percentages of Women Experiencing Miscarriage and Receiving Miscarriage Prevention Services by Disability Status and Type $(N=3,834)$

\begin{tabular}{|c|c|c|c|c|c|c|c|}
\hline & \multicolumn{2}{|c|}{ Disability status } & \multicolumn{5}{|c|}{ Disability type } \\
\hline & $\begin{array}{c}\text { No } \\
\text { disability }\end{array}$ & $\begin{array}{c}\text { Any } \\
\text { disability }\end{array}$ & Hearing & Vision & Cognitive & Physical & $\begin{array}{l}\text { Independent } \\
\quad \text { living }\end{array}$ \\
\hline $\begin{array}{l}\% \text { of women with any miscarriage in past } \\
5 \text { years }\end{array}$ & 21.83 & 31.63 & 41.66 & 27.23 & 33.14 & 33.32 & 32.86 \\
\hline$p$-value & & $<0.001$ & 0.004 & 0.213 & $<0.001$ & 0.033 & 0.030 \\
\hline $\begin{array}{l}\% \text { of women who received any services } \\
\text { to prevent miscarriage }\end{array}$ & 9.58 & 12.92 & 12.18 & 12.97 & 12.41 & 19.34 & 18.38 \\
\hline$p$-value & & 0.053 & 0.561 & 0.208 & 0.178 & 0.008 & $0.018 *$ \\
\hline $\begin{array}{l}\% \text { of women receiving each type of service } \\
\text { Bed rest }\end{array}$ & 33.98 & 51.67 & 44.58 & 64.54 & 45.53 & 67.47 & 65.00 \\
\hline$p$-value & & 0.030 & 0.562 & 0.018 & 0.225 & 0.007 & 0.032 \\
\hline Restricted physical activity & 50.81 & 57.95 & 42.55 & 53.00 & 58.49 & 86.72 & 62.39 \\
\hline p-value & & 0.398 & 0.664 & 0.901 & 0.463 & $<0.001$ & 0.447 \\
\hline Diagnosis of cause & 41.73 & 44.94 & 39.1 & 32.46 & 43.53 & 45.94 & 62.12 \\
\hline p-value & & 0.684 & 0.875 & 0.468 & 0.862 & 0.776 & 0.174 \\
\hline Drugs (e.g., progestin) & 42.42 & 39.05 & 29.73 & 38.31 & 46.46 & 22.74 & 43.81 \\
\hline$p$-value & & 0.705 & 0.599 & 0.746 & 0.697 & 0.142 & 0.958 \\
\hline
\end{tabular}

${ }^{a}$ Denominator is women who received at least one type of miscarriage prevention service $(n=327)$

$(p=0.0020)$. We found that miscarriages occurred at around 8 weeks of pregnancy for both women with and without disabilities, with no significant differences between groups (results not shown).

In our regression analyses examining adjusted odds of miscarriage and receipt of miscarriage prevention services, differences between women without and without disabilities persisted in all groups except for women with hearing disability. (Table 3). Women with cognitive disability $(\mathrm{aOR}=$ 1.86 [95\% CI: 1.32-2.61]) and women with physical disability (aOR $=1.99$ [95\%CI: $1.07-3.71])$ had the highest odds of miscarriage. Women with physical disability (aOR 2.82 [95\% CI $=1.46-5.45]$ ), or independent living disability $(\mathrm{aOR}=3.02[95 \% \mathrm{CI}=1.40-6.54])$ had the highest odds of

100

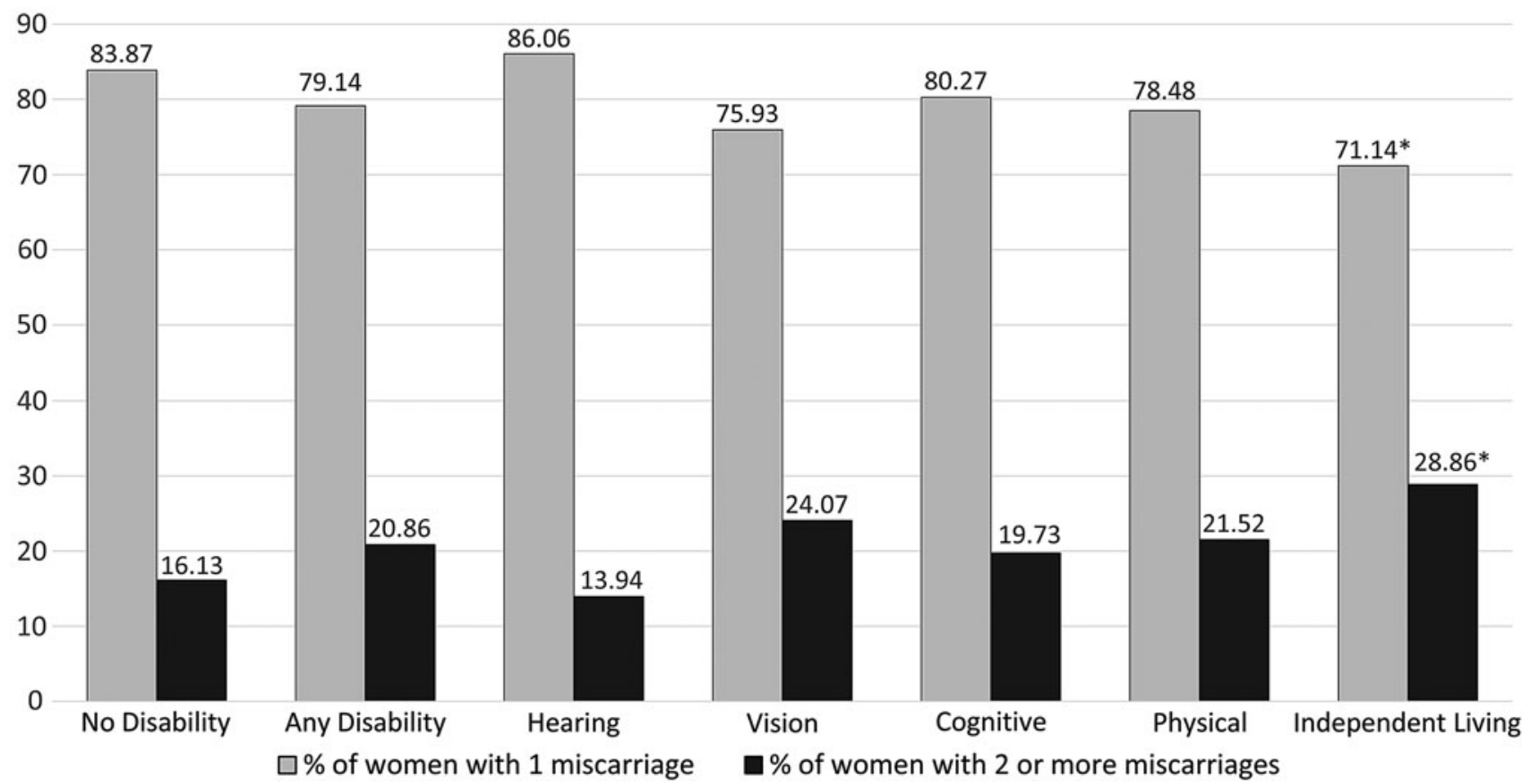

FIG. 2. Weighted percentages of women who had one miscarriage versus two or more miscarriages, among women who experienced miscarriage in the last five years $(n=831)$. *Indicates statistically significant difference $(p<0.05)$ from women without disabilities. 
35

$32.46^{*}$

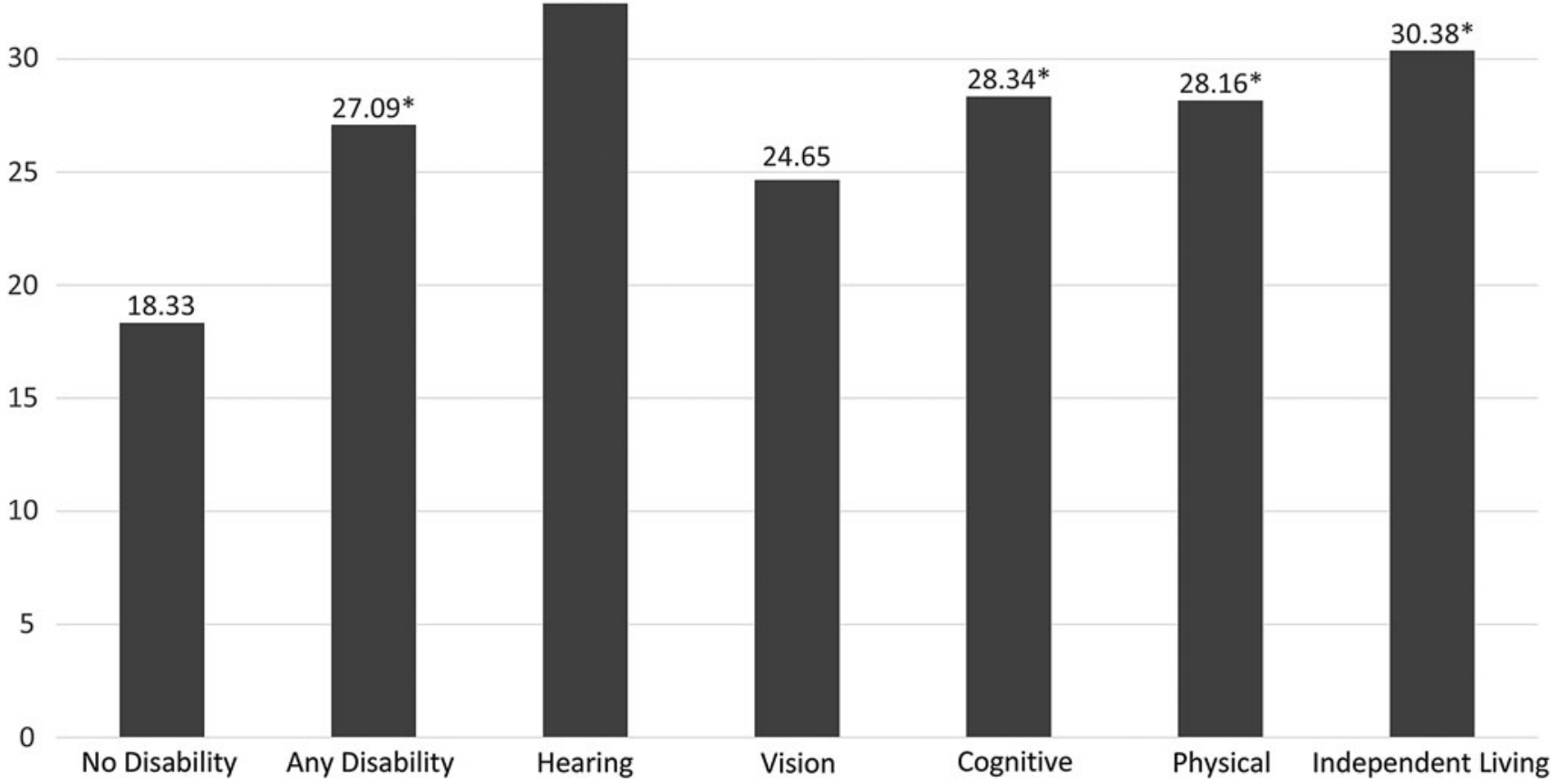

FIG. 3. Percentage of pregnancies ending in miscarriage, by disability status and type $(n=5,776)$. *Indicates statistically significant difference $(p<0.05)$ from women without disabilities.

receiving miscarriage prevention services. Our cell sizes were not large enough to assess associations between disability and the specific types of miscarriage prevention services in multivariable regression analyses.

\section{Discussion}

To our knowledge, this study is the first to examine miscarriage and medical care related to miscarriage among women with different types of disability. We found that even after adjustment for sociodemographic variables and prior history of miscarriage, women with any, cognitive, physical, or independent living disabilities were all more likely to have had at least one miscarriage in the last 5 years than women without disabilities. We also found that higher proportions of pregnancies among women with disabilities ended in miscarriage, suggesting that women with disabilities did not experience more miscarriage in the study period simply because they had more pregnancies.
While our bivariate analyses indicated that a high proportion of women with hearing disabilities experienced miscarriage in the last 5 years, we did not find this association to be statistically significant after covariate adjustment. However, this group was the smallest $(n=96)$ and it is likely that we were underpowered to detect a difference in this group. Only women with independent living disability were more likely to experience multiple miscarriages compared to women without disabilities. These results are consistent with and build on a prior finding that women with complex disabilities (which include difficulty with independent living activities) were more likely to have experienced miscarriage than women without disabilities. ${ }^{20}$

Because sporadic (as opposed to recurrent) miscarriage is not well-characterized in most women, it is difficult to determine why women with disabilities may be more likely to experience miscarriage. Other research has found that women with disabilities are more likely to have adverse preconception risk factors such as smoking, obesity, diabetes, asthma, frequent

Table 3. Adjusted Odds of Miscarriage and Miscarriage Prevention Services $(N=3,834)$

\begin{tabular}{lcc}
\hline Type of disability & Had at least one miscarriage in last 5 years & Received services to prevent miscarriage \\
\hline No disability & Ref & Ref \\
Any disability & $\mathbf{1 . 6 5}(\mathbf{1 . 2 1}, \mathbf{2 . 2 5})$ & $\mathbf{1 . 7 1}(\mathbf{1 . 2 0 ,} \mathbf{2 . 4 5})$ \\
Hearing disability & $2.16(0.98,4.74)$ & $1.34(0.56,3.21)$ \\
Vision disability & $1.31(0.75,2.30)$ & $1.69(0.81,3.52)$ \\
Cognitive disability & $\mathbf{1 . 8 6}(\mathbf{1 . 3 2}, \mathbf{2 . 6 1})$ & $\mathbf{1 . 8 2}(\mathbf{1 . 1 7}, \mathbf{2 . 8 1})$ \\
Physical disability & $\mathbf{1 . 9 9}(\mathbf{1 . 0 7}, \mathbf{3 . 7 1})$ & $\mathbf{2 . 8 2}(\mathbf{1 . 4 6}, \mathbf{5 . 4 5})$ \\
Independent living disability & $\mathbf{1 . 8 1}(\mathbf{1 . 0 5}, \mathbf{3 . 1 1})$ & $\mathbf{3 . 0 2}(\mathbf{1 . 4 0 , 6 . 5 4 )}$
\end{tabular}

Adjusted for maternal age, race, parity, prior history of miscarriage, current partner status, education, and health insurance.

Values in bold are statistically significantly different from women with no disabilities. 
mental distress, and lack of emotional support. ${ }^{27}$ Women with disabilities are also more likely to report complications during pregnancy, such as vaginal bleeding, kidney/bladder infections, stressful life events, and physical abuse during pregnancy. ${ }^{3}$ Many of these factors are on the pathway to adverse pregnancy outcomes such as preterm birth or low-birthweight, and they may also contribute to spontaneous miscarriage. ${ }^{21,22,28}$ The NSFG does not include information on medical conditions (e.g., diabetes) or life events that would contribute to perinatal health, so we were unable to adjust for these.

We found that women with elevated odds of miscarriage also had elevated odds of having received services to prevent miscarriage. Women with independent living disability had the highest odds of receiving miscarriage prevention services, which is consistent with our finding that women in this group were also the most likely to have experienced multiple miscarriages within the past 5 years. We were underpowered to assess adjusted associations between disability type and type of miscarriage prevention service received, but our bivariate analyses indicated that women with physical disability were much more likely than women without disabilities to have received recommendations to go on bed rest or limit their physical activity. While bed rest is commonly prescribed as a method for preventing miscarriage in women at elevated risk of miscarriage, such as women with threatened miscarriage (vaginal bleeding during pregnancy) or history of miscarriage, there is little evidence to suggest that bed rest is helpful. ${ }^{29,30}$

There is some evidence that providing luteal support via progestogen supplementation could prevent miscarriage, and that maternal serum markers have prognostic value in predicting miscarriage. ${ }^{30,31}$ While it is reassuring that across all disability groups, women with disability were about as likely to receive progesterone supplementation or diagnostic services as women without disabilities, the more frequent receipt of recommendations for bed rest or limiting physical activity is concerning. There are well-documented benefits to physical activity during pregnancy, including reducing the risk of cardiovascular and metabolic diseases and promoting psychological well-being. ${ }^{32}$ Further restricting the mobility of women who already have mobility limitations could be detrimental to their overall health, increasing risk of bone loss, muscle atrophy, and thrombus due to reduced circulation to lower extremities. ${ }^{30,32}$

While our study provides new information about miscarriage occurrence and receipt of miscarriage prevention services among women with disabilities, the cross-sectional nature of the NSFG limits our ability to make causal inferences. Although we restricted our study to pregnancies within the 5 years before interview, it is possible that even these relatively recent pregnancies may have preceded the onset of disability for some women in our sample. Misclassification of these women as having disabilities at the time of pregnancy would bias our results toward the null. Thus, we may have underestimated the associations between disability and miscarriage. On the other hand, it is possible that a small number of women may have acquired their disabilities through events that also contributed to miscarriage (e.g., car accident), in which case our results may be slight overestimates of the association between preexisting disability and miscarriage.

Additionally, while we did have information on how many pregnancy losses women had experienced when they first accessed services intended to prevent miscarriage, we did not have information on when women received any subsequent services, and many women received more than one service. In the follow-up questions, there was no information on the order of services or advice received (i.e., bed rest first, then testing, or vice versa). Therefore, we were unable to determine whether there were differences between groups in pregnancy outcomes following receipt of services.

Further, our results may be influenced by recall bias given the retrospective nature of the survey, though we lack existing research to suggest that women with disabilities are more or less likely to recall their pregnancy outcomes. Specifically in the case of women with cognitive disabilities, however, if they were less likely to remember their receipt of services related to miscarriage compared to women without disabilities, we would expect the presented estimate to be an underestimate of the true association between cognitive disability and miscarriage prevention services.

\section{Conclusions}

Our study found significant differences in occurrence of miscarriage and receipt of services to prevent miscarriage among women with different disabilities in a nationally representative sample. Additional research is needed to understand the reasons women with disabilities are more likely to have miscarriages. Future studies should incorporate the comorbidities and life stressors that women with disabilities disproportionally experience to determine what proportion of the association between disability and miscarriage can be attributed to these factors. Studies on miscarriage prevention services should prospectively evaluate the efficacy of these interventions in preventing miscarriage among women with disabilities. This information will be important to women with disabilities attempting to make informed decisions about their pregnancies.

\section{Author Disclosure Statement}

We declare that we have no real or perceived competing interests.

\section{Funding Information}

Research reported in this publication was supported by the Eunice Kennedy Shriver National Institute of Child Health and Human Development of the National Institutes of Health under award \#R21HD081309 (Horner-Johnson, PI). Support for Dr. Horner-Johnson's time was provided by grant \#K12HS022981 from the Agency for Healthcare Research and Quality (AHRQ) (Guise, PI). Dr. Darney was supported by R01HS025155 (Cottrell, PI) and AHRQ K12HS022981 (Guise, PI). The content is solely the responsibility of the authors and does not necessarily represent the official views of the National Institutes of Health or Agency for Healthcare Research and Quality. The funding agencies had no role in the conduct of the research or preparation of the article for submission.

\section{References}

1. Horner-Johnson W, Darney BG, Kulkarni-Rajasekhara S, Quigley B, Caughey AB. Pregnancy among US women: Differences by presence, type, and complexity of disability. Am J Obstet Gynecol 2016;214:529 e1-529 e9.

2. Iezzoni LI, Yu J, Wint AJ, Smeltzer SC, Ecker JL. Prevalence of current pregnancy among US women with and 
without chronic physical disabilities. Med Care 2013;51: 555-562.

3. Mitra M, Clements KM, Zhang J, Iezzoni LI, Smeltzer SC, Long-Bellil LM. Maternal characteristics, pregnancy complications, and adverse birth outcomes among women with disabilities. Med Care 2015;53:1027-1032.

4. Mitra M, Akobirshoev I, McKee MM, Iezzoni LI. Birth outcomes among U.S. women with hearing loss. Am J Prev Med 2016;51:865-873.

5. Akobirshoev I, Parish SL, Mitra M, Rosenthal E. Birth outcomes among US women with intellectual and developmental disabilities. Disabil Health J 2017;10:406-412.

6. Mitra M, Parish SL, Clements KM, Cui X, Diop H. Pregnancy outcomes among women with intellectual and developmental disabilities. Am J Prev Med 2015;48:300-308.

7. Parish SL, Mitra M, Son E, Bonardi A, Swoboda PT, Igdalsky L. Pregnancy outcomes among U.S. women with intellectual and developmental disabilities. Am J Intellect Dev Disabil 2015;120:433-443.

8. Gavin NI, Benedict MB, Adams EK. Health service use and outcomes among disabled Medicaid pregnant women. Womens Health Issues 2006;16:313-322.

9. Biel F, Darney B, Caughey A, Horner-Johnson W. Medical indications for primary cesarean delivery in women with and without disabilities. J Matern Fetal Neonatal Med 2019;18:1-8.

10. Darney BG, Biel FM, Quigley BP, Caughey AB, HornerJohnson W. Primary cesarean delivery patterns among women with physical, sensory, or intellectual disabilities. Womens Health Issues 2017;27:336-344.

11. Horner-Johnson W, Biel FM, Caughey AB, Darney BG. Differences in prenatal care by presence and type of maternal disability. Am J Prev Med 2019;56:376-382.

12. Horner-Johnson W, Biel FM, Darney BG, Caughey AB. Time trends in births and cesarean deliveries among women with disabilities. Disabil Health J 2017;10:376-381.

13. Morton C, Le JT, Shahbandar L, Hammond C, Murphy EA, Kirschner KL. Pregnancy outcomes of women with physical disabilities: A matched cohort study. PM R 2013;5: 90-98.

14. Brown HK, Kirkham YA, Cobigo V, Lunsky Y, Vigod SN. Labour and delivery interventions in women with intellectual and developmental disabilities: A population-based cohort study. J Epidemiol Community Health 2016;70: 238-244.

15. Mueller BA, Crane D, Doody DR, Stuart SN, Schiff MA. Pregnancy course, infant outcomes, rehospitalization, and mortality among women with intellectual disability. Disabil Health J 2019;12:452-459.

16. MacDonald SC, McElrath TF, Hernandez-Diaz S. Pregnancy outcomes in women with multiple sclerosis. Am J Epidemiol 2019;188:57-66.

17. Clements KM, Mitra M, Zhang J, Iezzoni LI. Pregnancy characteristics and outcomes among women at risk for disability from health conditions identified in medical claims. Womens Health Issues 2016;26:504-510.
18. Clements KM, Mitra M, Zhang J. Antenatal Hospital Utilization Among Women at Risk for Disability. J Womens Health (Larchmt) 2018;27:1026-1034.

19. Mitra M, Akobirshoev I, Moring NS, et al. Access to and satisfaction with prenatal care among pregnant women with physical disabilities: Findings from a national survey. J Womens Health (Larchmt) 2017;26:1356-1363.

20. Horner-Johnson W, Kulkarni-Rajasekhara S, Darney BG, Dissanayake M, Caughey AB. Live birth, miscarriage, and abortion among U.S. women with and without disabilities. Disabil Health J 2017;10:382-386.

21. Regan L, Rai R. Epidemiology and the medical causes of miscarriage. Baillieres Best Pract Res Clin Obstet Gynaecol 2000;14:839-854.

22. Rai R, Regan L. Recurrent miscarriage. Lancet 2006;368: 601-611.

23. Stirrat GM. Recurrent miscarriage. Lancet 1990;336:673-675.

24. Centers for Disease Control and Prevention. About the National Survey of Family Growth. Available at: www.cdc .gov/nchs/nsfg/about_nsfg.htm Accessed January 4, 2019.

25. Centers for Disease Control and Prevention. 2013-2015 National Survey of Family Growth (NSFG): Summary of design and data collection methods. Available at: https:// www.cdc.gov/nchs/data/nsfg/NSFG_2013-2015_Summary_ Design_Data_Collection.pdf Accessed January 4, 2019.

26. Dorsey R, Graham G. New HHS data standards for race, ethnicity, sex, primary language, and disability status. JAMA 2011;306:2378-2379.

27. Mitra M, Clements KM, Zhang J, Smith LD. Disparities in adverse preconception risk factors between women with and without disabilities. Matern Child Health J 2016;20: 507-515.

28. Lindbohm ML, Sallmen M, Taskinen H. Effects of exposure to environmental tobacco smoke on reproductive health. Scand J Work Environ Health 2002;28 Suppl 2: 84-96.

29. Aleman A, Althabe F, Belizan J, Bergel E. Bed rest during pregnancy for preventing miscarriage. Cochrane Database Syst Rev 2005:2;CD003576.

30. Qureshi NS. Treatment options for threatened miscarriage. Maturitas 2009;65 (Suppl 1):S35-S41.

31. Norwitz ER, Caughey AB. Progesterone supplementation and the prevention of preterm birth. Rev Obstet Gynecol 2011;4:60-72.

32. Durstine JL, Painter P, Franklin BA, Morgan D, Pitetti KH, Roberts SO. Physical activity for the chronically ill and disabled. Sports Med 2000;30:207-219.

Address correspondence to: Willi Horner-Johnson, PhD Institute on Development and Disability Oregon Health \& Science University 707 SW Gaines Street Portland, OR 97239

E-mail: hornerjo@ohsu.edu 